\title{
Special volume on 'Recent Developments in Queueing Theory' of the third ECQT conference
}

\author{
Ivo J. B. F. Adan ${ }^{1} \cdot$ Bernardo D'Auria ${ }^{2}$ Offer Kella ${ }^{3}$ \\ Published online: 3 September 2019 \\ ๑) Springer Science+Business Media, LLC, part of Springer Nature 2019
}

This volume is dedicated to work selected from the 74 presentations at the third European Conference on Queueing Theory, held in Jerusalem, Israel, July 2-4, 2018. The six papers in this special issue have been chosen, revised and edited for publication through a careful refereeing process. There will be a second, companion volume, with a few more papers, shortly. QUESTA also generously published special issues of papers selected from the first and second ECQT meetings (vol. 82, issues 1-2, 2016; vol. 87, issues 3-4, 2017; and vol. 88, issues 1-2, 2018). These special issues would not have been possible without the Technical Program Committee of ECQT, the expert reviewers and the support and advice of Editor-in-Chief Sergey Foss.

The topics considered in each of the articles of this special issue are quite diverse and, in fact, are all different. The sub-areas to which they belong can be categorized as queueing games, weak convergence (of layered queueing systems), simulation, redundancy scheduling (in queues), regenerative theory (and applications), and coupling and stochastic ordering (in a symmetric join the shortest queue model).

Bountali and Economou consider a Markovian batch service system comprised of two nodes in tandem: a batch assembly node followed by a single server node. Customers behave strategically: Observing the number in the batch assembly node upon arrival they decide to join or not. The authors characterize the customer equilibrium strategies and explore the qualitative properties of the strategic customer behavior through numerical experiments.

\footnotetext{
$凶 \quad$ Offer Kella

offer.kella@gmail.com

Ivo J. B. F. Adan

i.adan@tue.nl

Bernardo D'Auria

bernardo.dauria@uc3m.es

1 Department of Industrial Engineering and Innovation Sciences, Eindhoven University of Technology, P.O. Box 513, 5600 MB Eindhoven, The Netherlands

2 Department of Statistics, Madrid University Carlos III, 28903 Getafe, Madrid, Spain

3 Department of Statistics and Data Science, The Hebrew University of Jerusalem, 9190501 Jerusalem, Israel
} 
Buijsrogge, de Boer and Scheinhardt study non-Markovian queues in tandem. They estimate the probability that the number of customers in the system reaches some given level during a busy cycle, employing importance sampling simulation. The authors propose a state-dependent change of measure and prove that this change of measure is asymptotically efficient, provided all supports of service time and inter-arrival time distributions are bounded and some technical conjecture holds.

Raaijmakers, Borst and Boxma consider redundancy scheduling in parallel-server systems with non-exponential services. In particular, they focus on the stability condition for the cancel-on-completion policy. A simple sufficient stability condition is derived for general services, and this condition is proved to be asymptotically nearly necessary for the special case of scaled Bernoulli service requirements when the scale parameter grows large.

Aveklouris, Vlasiou and Zwart are motivated by an electrical vehicle (EV) charging problem which is modeled as a layered queueing model. In particular, in the first layer, queueing is not allowed (Erlang loss model) and the number of EVs in the charging facilities is the number of occupied charging stations. At any one time, there is a cap on how many vehicles may be charged at a time which is typically smaller than the total number of available charging stations. The randomness in this model arises from the inter-arrival times, parking times and charging times, all of which are assumed independent (and i.i.d. within each type). The most interesting performance measure the authors are after is the fraction of vehicles that get fully charged. For this purpose, with the exception of a simple case where exact results are possible, the authors prove fluid and diffusion approximations and apply them.

Jacobovic and Kella prove (under some conditions) asymptotic independence for a vector of possibly dependent regenerative processes with a certain dependence structure. The results are motivated by and then applied to various unrelated queueing and storage models.

Tibi introduces a variant of the symmetric join-the-shortest-queue model by assuming one service rate for the shortest queue and another for the longest queue. Through coupling arguments, she shows that the total number in the system is stochastically ordered as the service rate of the longest queue varies. For the case of finite capacity queues, she derives, among others, the Laplace transform of the time to overflow and an explicit expression for the stationary blocking probability through the use of martingales and hitting times.

Publisher's Note Springer Nature remains neutral with regard to jurisdictional claims in published maps and institutional affiliations. 